\title{
Genetic Algorithms: A Tool for Image Segmentation
}

\author{
Alaa Sheta \\ Computer Science Department \\ WISE University \\ Amman 11947, Jordan \\ Email: asheta66@gmail.com
}

\author{
Malik S. Braik \\ Electronic, Electrical and Computer Eng. Dept. \\ University of Birmingham \\ Birmingham, Edgbaston, UK \\ Email: MSB158@bham.ac.uk
}

\author{
Sultan Aljahdali \\ Department of Computer Science \\ Taif University \\ Taif, Saudi Arabia \\ Email: aljahdali@tu.edu.sa
}

\begin{abstract}
Genetic Algorithms (GAs) are increasingly being explored in many areas of image analysis to solve complex optimization problems. They rapidly gained acceptance in the scientific community as powerful statistical search method whicj allows us to consider the segmentation problem as an optimization problem. In this paper, we propose the use of GAs in an integrated manner with traditional image segmentation techniques to provide an efficient segmentation and edges detection for selected natural images. The developed experimental results are compared with the results of other known existing segmentation algorithms such as K-mean clustering, and global threshold methods. The proposed method is capable of achieving a satisfactory results. Accordingly, the GAs based image segmentation method will definitely help in solving various complex image processing tasks.
\end{abstract}

Keywords-thresholding, image segmentation, genetic algorithms, edge detection, optimization

\section{INTRODUCTION}

Image segmentation is an essential step for many advanced imaging applications, and considered one of the hard problems of image recognition and understanding. In case of analyzing and understanding the information in an acquired image, it is necessary to extract the area in which the objective material is included. Moreover, depending on the problem domain or application, there are several types of images that could be processed and analyzed such as, light intensity (i.e. gray scale), color, range, Infra-Red (IR) [1] and SAR images [2].

Many range image segmentation algorithms was proposed in [3]. These segmentation algorithms were tuned using number of heuristic algorithms such as GAs to provide exact results on a given group of images [4]. Segmentation methods frequently use thresholds to decide whether two pixels are in the same region or not. However, the thresholds negatively affect the image segmentation results often in accuracy. Therefore, the tuning values of the thresholding algorithms must be set up carefully [3], [5]. A multilevel thresholding method which allows the determination of the appropriate number of thresholds as well as the adequate threshold values was proposed in [5]. This method combines both genetic algorithm and wavelet transform. In [6], authors presented a new distributed image segmentation algorithm structured as a multiagent system

A. F. Sheta is a Professor of Computer Science and Engineering, Computer Science Department, The World Islamic Science and Education (WISE) University, Amman, Jordan. He is on leave from the Electronics Research Institute (ERI), Cairo, Egypt composed of a set of segmentation agents and a coordinator agent.

Despite the large number of segmentation techniques presently available, no general methods admit a unique solution because contest may take part in the decision phase. It follows that general solutions are not possible, and each proposed technique can be used only to solve class of problems [7]. Consequently, computing such an image partition is a very high complexity problem, hence, development of a unified method to image segmentation is still an open research area which significantly in need for more effort [8].

This research proposed a new method for image segmentation problem using Genetic Algorithms (GAs). Image segmentation problem is formulated as an optimization problem based GAs. The contribution of this work is the use of GA to build a reliable and accurate method to tune a set of parameters for a traditional filter. One reason for using this kind of method is mainly related with the GA ability to deal with complex search spaces in situations where only minimum knowledge is available. Moreover, the proposed GA method for image segmentation is compared with other traditional methods in the literature. The applicability of the proposed methods has been tested on a large class of images; the obtained results are encouraging, performing well in all of the proposed problems.

\section{WHAT IS GAS?}

Genetic algorithms (GAs) are adaptive search procedures which were first introduced by Holland [9], and extensively studied by Goldberg [10], De Jong [11-13], and others. One of the most successful areas of application has been the use of GAs to solve a wide variety of difficult numerical optimization problems. GAs complement existing optimization methodes nicely in that they require no gradient information and are much less likely to get trapped in local minima on multimodal surfaces. In addition, GAs have been shown to be quite insensitive to the presence of noise [14].

- In GAs, each possible solution within the population of a biological individual is coded in so called "chromosome" (i.e. individual). A number of chromosomes generate what is called a "population". The structure for each individual can be represented as a string of characters which are usually binary digits [9] or real numbers. The chromosomes share data with other, and each chromosome is assigned a fitness score according to how good a 
solution to the problem based on a given fitness function. The solutions are taken according to their fitness values and used to construct new solutions by a hope that the new solutions will be better than the old solutions and a generation is complete. Thus, the whole population moves like a one group towards an optimal area [9], [10], [15], [16].

- At each generation, each individual is evaluated and recombined with others on the basis of its fitness. The expected number of times an individual is selected for recombination is proportional to its fitness; the fitness is relative to the rest of the population. New individuals are created using crossover and mutation operations.

- Crossover operates by selecting a random location in the genetic string of the parents (crossover point) and concatenating the initial segment of one parent with the final segment of the second parent to create a new child. A second child is simultaneously generated using the remaining segments of the two parents [15], [16].

- Mutation provides for occasional disturbances in the crossover operation by inverting one or more genetic elements during reproduction [9], [15].

\section{Image Segmentation Using GAS}

GA is proposed to explore the solution space, in the sense that each pixel is grouped into other pixels by distance function based upon both local and global already computed segments. There are two basic issues needed to be addressed in designing a GA for image segmentation, they are:

- 1) selecting an appropriate representation for each potential solution;

- 2) formulating an appropriate fitness functions in terms of the weight.

Almost every image segmentation algorithm contains parameters that are used to control the segmentation results; the genetic system can dynamically change the parameters to achieve the best performance. The image segmentation based GAs process may be summarized as follows:

- Once the initial population is available, the genetic cycle begins as shown in Figure 1.

- The quality of the segmented results for each parameter set is then evaluated. If the maximum segmentation quality for the current population is above a predefined threshold value or another stopping criterion, then, the cycle will terminate. Alternatively, if the performance is not achieved, the genetic recombination operators (crossover and mutation) are applied to the high strength individuals in the population, which yield a new set of offspring that has better performance.

- The new population is supplied back to the image segmentation process, and thus, the cycle begins again. Each pass through the loop (Segmentation-Fitness-Crossover, and Mutation) is known as generation. The cycle shown in Figure 1 continues until the maximum fitness or termination criteria are achieved.

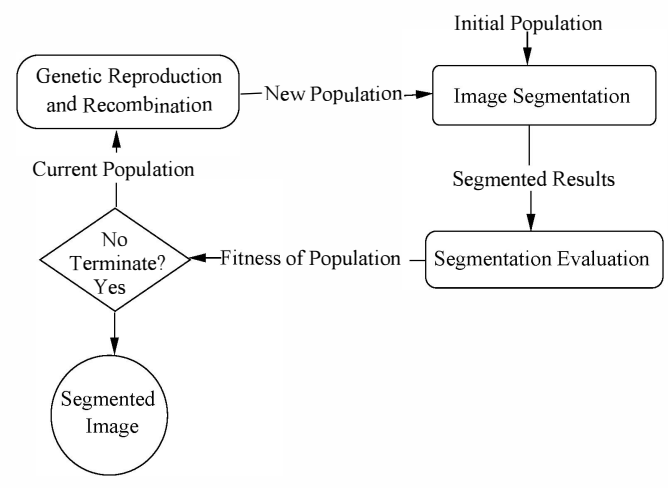

Fig. 1. Flow chart of the GAs based segmentation method

- The fitness function is related to the internal variance of each image segment, and has two weights for grouping the pixels into subsets based on the distance function. Hence, the pixel intensity and spatial position are used, Therefore, the distance function is based on the pixel intensity and spatial position to define the similarity between pixels as shown in Figure 2.

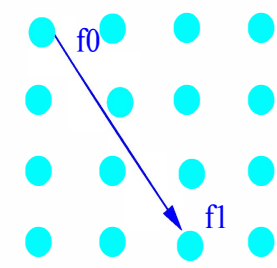

Fig. 2. The graph and the distance function

\section{Proposed Segmentation Algorithm}

The segmentation of a digital image $F$ can be considered as a natural partitioning of its pixels, based on a distance function that is defined in $F$. $F$ of size ( $M \times N$ pixels) can be described as:

$$
F=F(i, j), 1 \leq i \leq M, 1 \leq j \leq N, 0 \leq F(i, j) \leq C
$$

where $C$ is the number of class prototypes that can be selected freely, so that, $F$ is a collection of pixels where each pixel value is proportional to the degree of region similarity. The pixel values in $F$ are normalized in the range $[0,1]$. Pixels with values close to one are good candidates for being considered as edge points. The regions or subsets of $F$ are denoted by: $S=\left(S_{1}, \ldots, S_{K}\right)$.

\section{A. Image Preprocessing}

The preprocessing phase of image segmentation includes image similarity based on the distance function, connected component labeling, edge tracing, and initial fitness evaluation. The regions with similar pixels are grouped into regions as a final result of segmentation or clustering. At the end of 
clustering procedure, a label is assigned to each pixel that take a value between 1 and $K$, such that, each possible region of $F$ can be represented by a matrix, and it corresponds to a pixel of the subset or partition. $F$ is associated to the weighted graph $G$ as: $G \equiv<F, \delta>. \delta$ is the distance function that links between $f_{0}$ and $f_{1}$ as shown in Figure 2 .

\section{B. Image Partitioning}

The segmentation algorithm depends on partition the vertices of the weighted graph $G=(V, E, \delta)$ into $K$ subsets. An example of weighted graph of three segments is shown in Figure 3. As given previously, $G=(V, E, \delta), S_{i} \bigcap S_{j}=\Phi$,

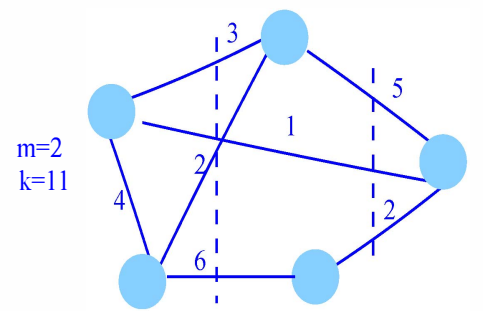

Fig. 3. Image segmentation and partitioning the graph

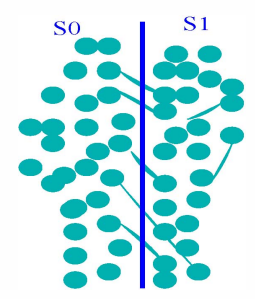

Fig. 4. Illustration the bipartition process between two subsets

and $S_{i} \cup S_{j}=F$. Hence, we want to disjoint $S_{i}$ and $S_{j}$ by removing edges connecting the two parts as shown in Figure 4). The cut of $S_{i}$ and $S_{j}$ can be defined as given in Equation 2:

$$
\operatorname{cut}\left(S_{i}, S_{j}\right)=\sum_{i \in S_{i} j \in S_{j}} \delta(i, j)
$$

The optimal partition is the one that minimizes the distance function (similarity function). A pixel $f \in F$ is represented in a $3 D$ feature space with $\left(i_{f}, j_{f}, l_{f}\right)$. $(i, j)$ represents the pixel position, and $l$ represents its gray level.

In this research, the grouping of pixels into partitions is based on the distance function computed for each partition as defined in Equation 3.

$$
\begin{aligned}
\delta(i, j) & =w_{0} \times \frac{\left|l_{i}-l_{j}\right|}{\max \left(l_{i}, l_{j}\right)} \\
& +w_{1} \times \frac{1}{|N(i)|} \sum_{z \in N(i)} \frac{\left|l_{z}-l_{j}\right|}{\max \left(l_{z}, l_{j}\right)}
\end{aligned}
$$

$w_{0}, w_{1} \geq 0$ and $\forall i, j \in F, i \sim j \Longleftrightarrow \delta(i, j) \leq \Phi$. $N(i)$ is a neighborhood of the pixel $i . w_{0}$ and $w_{1}$ are the weights attached to its feature. Therefore, the ranges of $w_{0}, w_{1}$ are normalized between 0 and 1 , consequently, each feature contributes equally to the distance function.

\section{EXPERIMENTAL SETUP FOR GAs}

For an input image $F$ of size $N \times M, F(i, j)$ indicates to the gray-level value of the pixel located in $(i, j)$ location. The proposed procedure of image segmentation based GAs consists of the following steps:

\section{A. GA Data coding}

The genetic chromosome is coded by a 32 bit binary string. The 8 least significant bits identifies the pixel label of the cluster to which the pixel belongs after the clustering procedure. While, the pixel position $(i, j)$ is coded into the 24 most significant bits. Consequently, each chromosome can be denoted with the order pair as shown in Figure 5.

$$
\begin{array}{|l|l|}
\hline b_{31}, \ldots \ldots, b_{8} & b_{7}, \ldots, b_{0} \\
\hline
\end{array}
$$

Fig. 5. A Chromosome representation

\section{B. Fitness Function}

Evaluation a segmentation technique is not an easy task, since the expected segmentation result depends on the application. The fitness function of the segmentation quality varies from image to image. Therefore, the definition of the objective function itself can be subject of debate because there are no single, universally accepted measures of segmentation performance available [7], [17], [18].

The fitness function has been defined on the basis of the distance function computed between each chromosome and the corresponding region $S$. The fitness function has been defined with two weights characterized by $w_{0}$ and $w_{1}$. In this study, an agreement indicator (i.e. fitness function) between human and automatic classification has been defined as given in Equation 4 [19]:

$$
\gamma=\frac{1}{K} \sum_{k=1}^{K} \beta_{k} \times \frac{\# a g r_{k}}{\bar{P}_{k}}
$$

where: $\left\{\bar{P}_{k}=\max \left(\left|\operatorname{Seg}_{k}\right|,|S|\right)\right\}, \operatorname{Seg}_{k}$ denotes the $k^{\text {th }}$ segment retrieved by humans, $S$ denotes the $k^{\text {th }}$ segment retrieved by the machine, $\left|S e g_{k}\right|$ and $|S|$ denote the corresponding size and $\# a g r_{k}$ is the largest pixel intersection between $S e g_{k}$ and $S$.

\section{Crossover and Mutation}

The classical single point crossover with crossing probability $P_{c}$ and bit mutation with probability $P_{m}$ have been evolved in the proposed genetic system. An example of single point crossover and bit mutation is shown in Figure 6. 


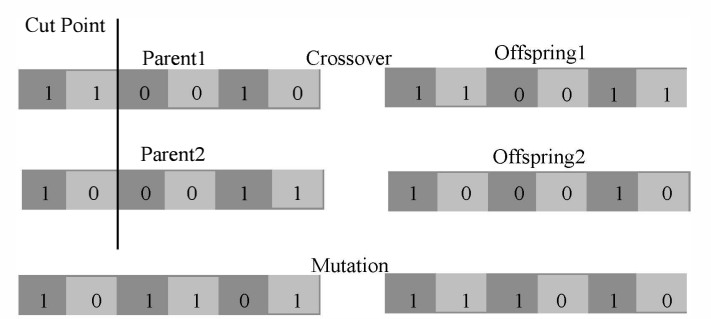

Fig. 6. Example of crossover and mutation operations

\section{Selection Process}

In the GAs poputaion, $N \times M$ numbers of chromosomes are generated using random numbers, such that:

$$
P(0)=\left\{\nu(0), \nu(1), \ldots, \nu_{M \times N}(0)\right.
$$

$P(0)$ is the population at time 0 . Then, the single point crossover and bit mutation are applied to the initial population to get another generation, $P(1)$, and so on. The new population is determined by the selection process such that the new population is better than the old population in terms of its fitness.

\section{E. Termination Condition}

The procedure from step $a$ to step $d$ is repeated until the convergence condition is satisfied or the number of generations is reached. The convergence process is based on the total variance of the region as shown in Equation 6.

$$
\operatorname{Var}_{t}=\sum_{k=1}^{K} \sigma(k)^{2}
$$

where, $t$ is the iteration number, $\sigma^{2}$ is the grey-level variance of region $k$, and $K$ is the total number of regions. The loop continue until $\left|V a r_{t+1}-\operatorname{Var}_{t}\right| \leq \epsilon$, where $\epsilon$ is a small defined value. The GA based image segmentation proposed method is shown in Figure 7.

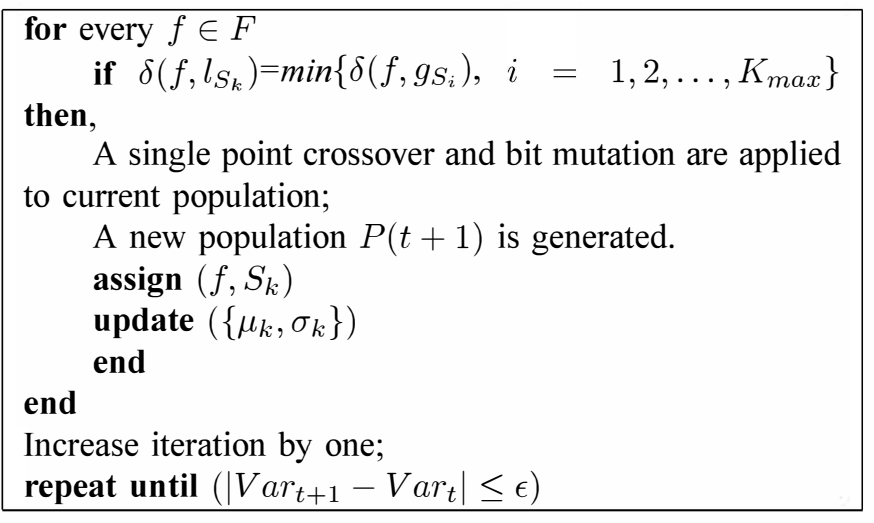

Fig. 7. GA based image segmentation algorithm

\section{EXPERIMENTAL RESULTS}

The proposed segmentation algorithm has been implemented using MATLAB with Signal Processing Toolbox and Statistics Toolbox under windows system. Several experiments are tested to demonstrate the performance of the proposed segmentation algorithm. The setting parameters of the GAs run are shown in Table I. A set of four images are used to illustrate the usability of the proposed algorithm as seen in Table II. The proposed segmentation problem based GAs is treated

TABLE I

TUNING PARAMETERS OF GAS

\begin{tabular}{|l|c|}
\hline Parameter & Value \\
\hline Population size & 50 individuals \\
\hline Gene length & 32 bits \\
\hline Maximum generation & 200 \\
\hline The Prob. of Mutation $P_{m}$ & 0.01 \\
\hline The Prob. of Crossover $P_{c}$ & 0.8 \\
\hline
\end{tabular}

TABLE II

IMAGE SIZES AND NUMBER OF GENERATIONS FOR GA RUNS

\begin{tabular}{|l|c|c|}
\hline Image & size & iterations \\
\hline Aish & $383 \times 801$ & 200 \\
\hline Hand & $243 \times 909$ & 200 \\
\hline House & $192 \times 765$ & 200 \\
\hline Fish & $74 \times 390$ & 200 \\
\hline
\end{tabular}

as a partitioning problem. It has been applied to polygons, natural, and texture images. The results show good agreement between natural and automatic partitioning. The experimental results are compared with the results of other known existing segmentation algorithms such as K-mean clustering [20], and global threshold methods [21]. Segmentation results are shown in Figures 8, 9, 10, and 11. It can be seen that the GA proposed method obtains the most optimal results, but with high computational effort.

The GAs convergence has been achieved in all experiments. Figure 12 shows the convergence of the segmentation algorithm in the case of hand image. Figure 12 indicates that the proposed segmentation method based GAs converges to a solution near the global optimum. The agreement indicators of the tested images are shown in Table III.

TABLE III

AGREEMENT INDICATORS OF THE TESTED IMAGES

\begin{tabular}{|l|c|c|c|c|}
\hline Method $/ \gamma$ & Aish & Hand & House & Fish \\
\hline K-Mean & 0.69 & 0.66 & 0.61 & 0.63 \\
\hline Global & 0.59 & 0.53 & 0.55 & 0.49 \\
\hline Proposed GA-Method & 0.75 & 0.83 & 0.80 & 0.83 \\
\hline
\end{tabular}

\section{COMPARISON WITH OTHER TECHNIQUES}

For illustrating the efficiency of the proposed edge detection method, the results are compared to other standard existing edge detection methods. 

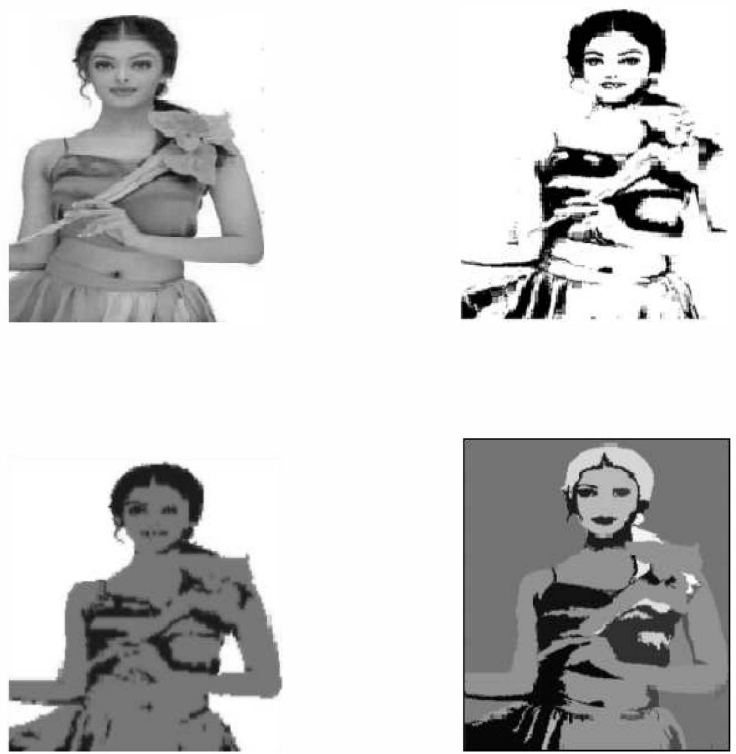

Fig. 8. Results of segmentation: upper left Aish image; upper right Global threshold; lower left K-Means clustering method; lower right GAbased method;
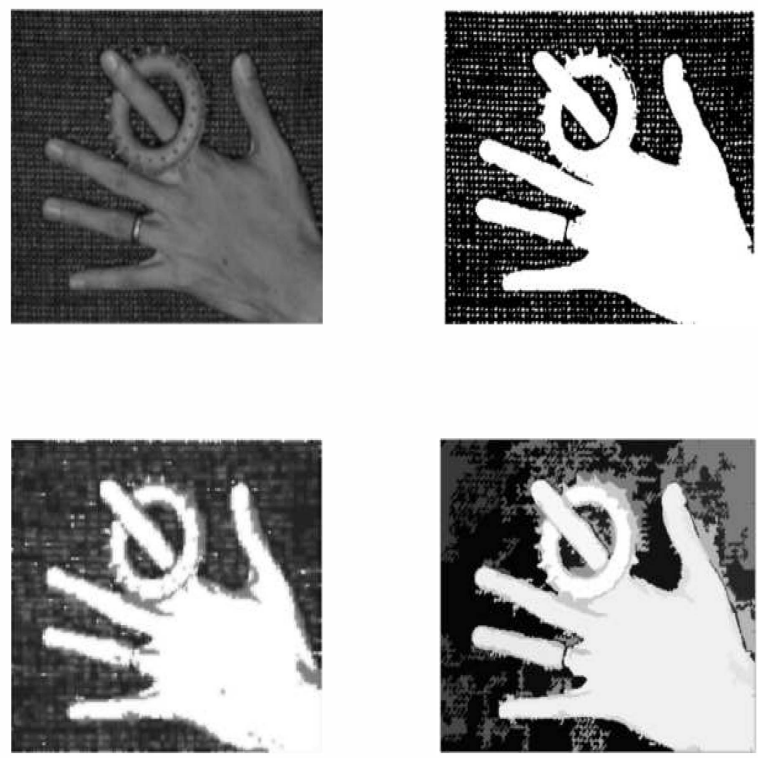

Fig. 9. Results of segmentation: upper left - Hand image; upper right - Global threshold; lower left - K-Means clustering method; lower right - GAbased method;

\section{A. Sobel}

The Sobel operator approximates the first derivative that performs a spatial gradient measurement on the image, and so, emphasizes on regions of high spatial frequency that correspond to edges. Sobel operator consists of a pair of $3 \times 3$ convolution kernels. The kernels are designed to respond maximally to edges running vertically and horizontally, one kernel for each of the two perpendicular orientations. Thus, the operator has a problem of identifying edges in three dimensions as seen in Figures 13 and 15. Furthermore, the
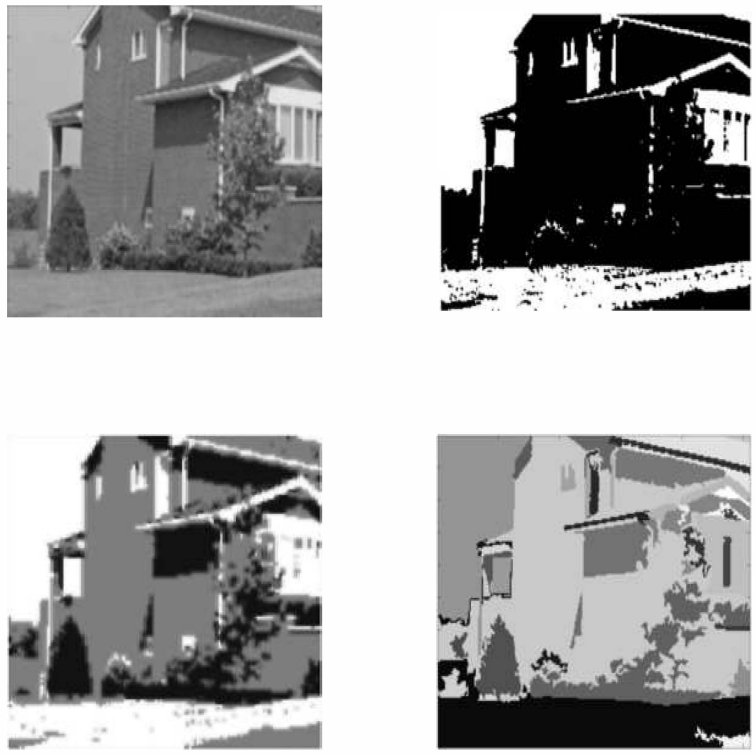

Fig. 10. Results of segmentation: upper left - House image; upper right - Global threshold; lower left - K-Means clustering method; lower right GAbased method;
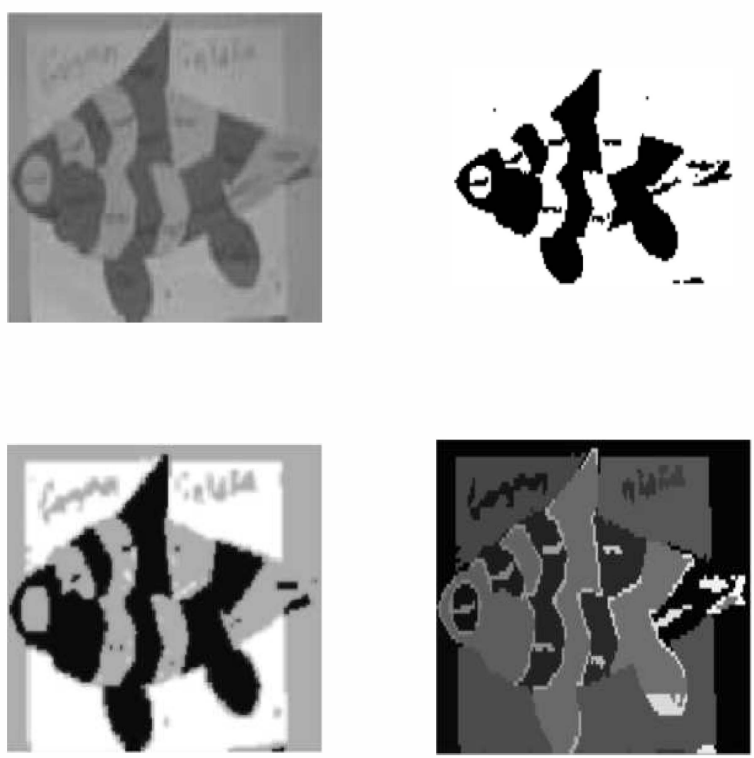

Fig. 11. Results of segmentation: upper left - Fish image; upper right - Global threshold; lower left - K-Means clustering method; lower right - GAbased method;

operator needs a threshold value which negatively effects on the result of the edges detection (see Table IV).

\section{B. Prewitt}

The Prewitt operator is similar to the Sobel operator. It approximates the first order derivative, but uses slightly different kernels. The operator consists of a pair of $3 \times 3$ convolution kernels. The kernels are designed to respond maximally to edges running vertically and horizontally. Prewitt operator needs a threshold value and is very sensitive to noise as seen in Figure 14. Therefore, the operator is not effective to the 


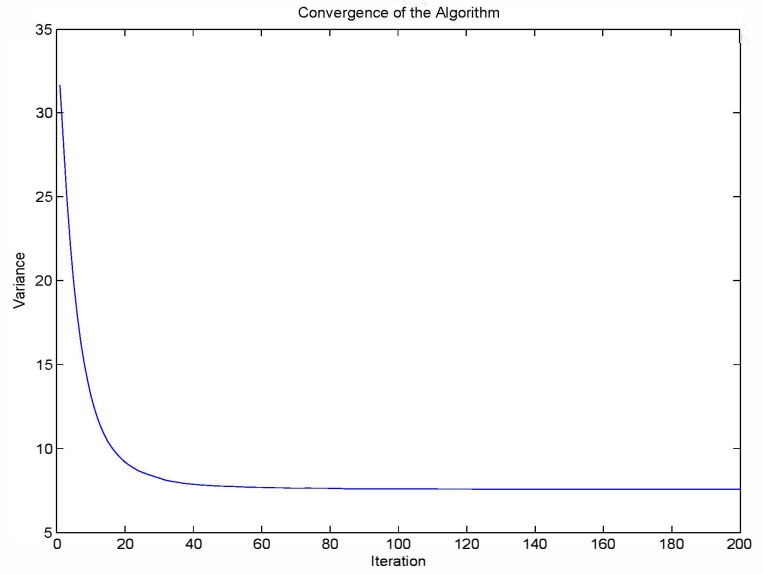

Fig. 12. Convergence of GAs via the number of gerenations: The hand image

detection of the edges.

\section{Roberts}

The Roberts cross operator performs a simple spatial gradient measurement on the image. It thus highlights regions of high spatial frequency which often corresponds to edges. The operator consists of a pair of $2 \times 2$ convolution kernels. The kernels are designed to respond maximally to edges running vertically and horizontally, one kernel for each of the two perpendicular orientations. Consequently, only four input pixels are needed to be examined to determine the value of each output pixel, and only subtractions and additions are used in the calculation.

Robert operator is very sensitive to noise since it uses such a small kernel, and operator produces very weak responses to genuine edges unless they are very sharp as seen in Figures 13, 15, and 16. Also, the operator needs a threshold value which negatively effects on the result of edges detection. The threshold values of the operators in the case of aish image are shown in Table IV.

TABLE IV

THE THRESHOLD VALUES IN THE CASE OF AISH IMAGE

\begin{tabular}{|c|c|}
\hline Operator & Threshold value \\
\hline Sobel & 0.075000 \\
\hline Prewitt & 0.075000 \\
\hline Robert & 0.215700 \\
\hline
\end{tabular}

The parameter values of the GAs run for the edge detection problem are shown in Table V.

TABLE V

Tuning Parameters of GAs For the EDge Detection PROBlem

\begin{tabular}{|l|c|}
\hline Parameter & Value \\
\hline Population size & 50 \\
\hline Gene length & 32 \\
\hline Maximum generation & 200 \\
\hline The probability of mutation $P_{m}$ & 0.01 \\
\hline The probability of crossover $P_{c}$ & 0.8 \\
\hline
\end{tabular}
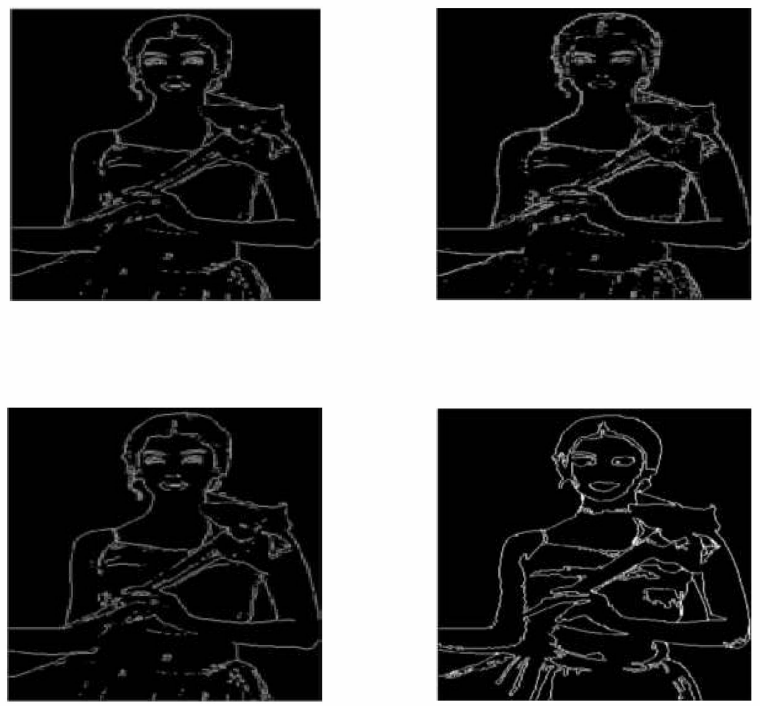

Fig. 13. Results of edge detection of the aish image: upper left - Prewitt operator; upper right - Robert operator; lower left - Sobel operator; lower right - GA based method;
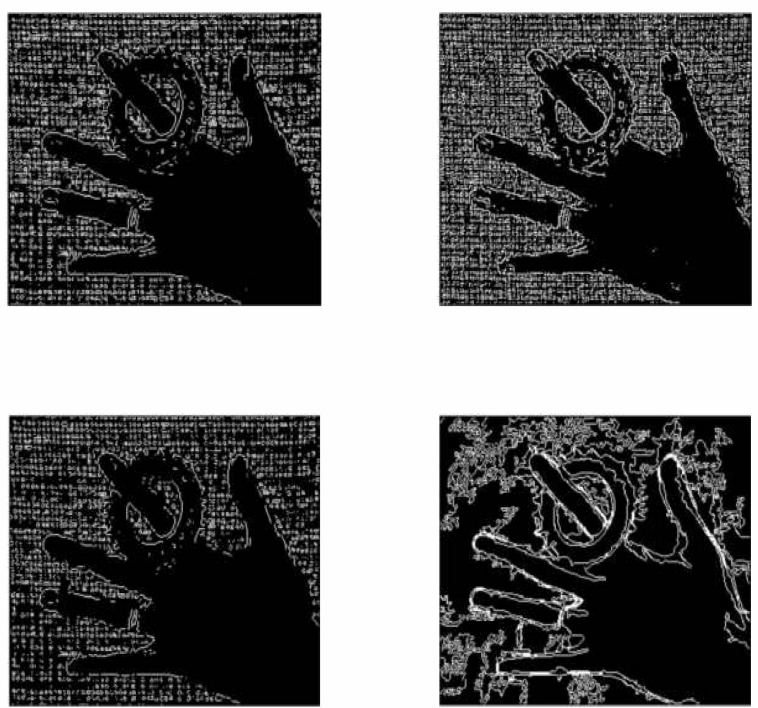

Fig. 14. Results of edge detection of the hand image: upper left - Prewitt operator; upper right - Robert operator; lower left - Sobel operator; lower right - GA based method;

\section{CONCLUSIONS AND FUTURE WORKS}

In this paper, we used GAs to solve the image segmentation problem. The problem was treated as an optimization problems based GA. GAs was used to segment an image by using an optimization function without any threshold values. GAs based image segmentation can provide more accurate results than traditional segmentation methods. The genetic procedure provided a faster convergence to the optimal solution. This is because the sampling strategy allows exploring the solution space by a strategy that is not biased. The preliminary results indicate that GA-based methods outperform the traditional methods in terms of quality. Finally, the obtained results of image segmentation and edges detection indicate that the 

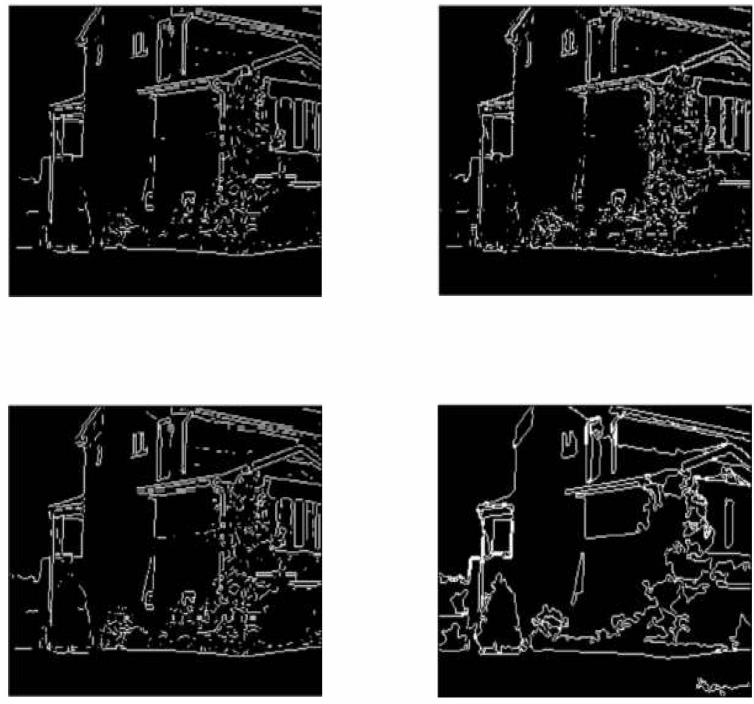

Fig. 15. Results of edge detection of the house image: upper left - Prewitt operator; upper right - Robert operator; lower left - Sobel operator; lower right - GA based method;
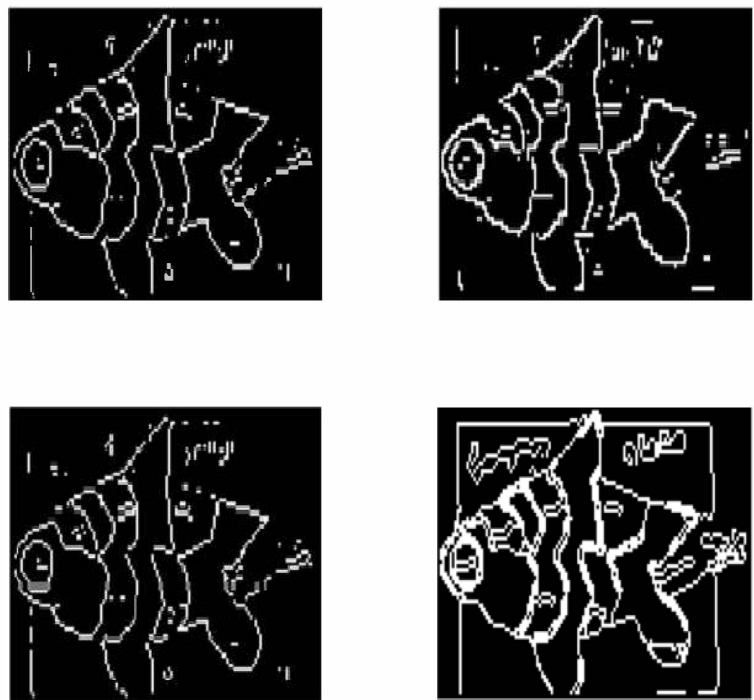

Fig. 16. Results of edge detection of the fish image: upper left - Prewitt operator; upper right - Robert operator; lower left - Sobel operator; lower right - GA based method;

proposed methodes have a high effectiveness on a large category of images applications. An extension work will focus on developing hybrid algorithms such as GAs and Artificial Neural Networks (ANNs) in order to reduce the processing time and increase the visual quality of the final segmentation underscoring the advantages of hybrid algorithms.

\section{REFERENCES}

[1] A. Ajlouni and A Sheta, Image Processing Techniques for Landmine: A Framework for Detection and Classification. LAP LAMBERT Academic Publishing, ISBN 978-3-8473-3721-8, 2012.

[2] G. Gao, L. Zhao, J. Zhang, D. Zhou, and J. Huang, "A segmentation algorithm for sar images based on the anisotropic heat diffusion equation," Pattern Recogn., vol. 41, pp. 3035-3043, Oct. 2008.
[3] G. Pignalberi, R. Cucchiara, L. Cinque, and S. Levialdi, "Tuning range image segmentation by genetic algorithm," EURASIP J. Appl. Signal Process., vol. 2003, pp. 780-790, Jan. 2003.

[4] S. Chabrier, C. Rosenberger, B. Emile, and H. Laurent, "optimizationbased image segmentation by genetic algorithms," J. Image Video Process., vol. 2008, pp. 10:1-10:10, Jan. 2008.

[5] K. Hammouche, M. Diaf, and P. Siarry, "A multilevel automatic thresholding method based on a genetic algorithm for a fast image segmentation," Comput. Vis. Image Underst., vol. 109, pp. 163-175, Feb. 2008.

[6] K. E. Melkemi, M. Batouche, and S. Foufou, "A multiagent system approach for image segmentation using genetic algorithms and extremal optimization heuristics," Pattern Recogn. Lett., vol. 27, pp. 1230-1238, Aug. 2006.

[7] A. Brink, "Using spatial information as an aid to maximum-entropy image threshold selection," PRL, vol. 17, pp. 29-36, January 1996.

[8] S. M. Bhandarkar and H. Zhang, "Image segmentation using evolutionary computation," IEEE Transactions on Evolutionary Computation, vol. 3, no. 1, pp. 1-21, 1999

[9] J. Holland, Adaptation in Natural and Artificial Systems. Ann Arbor, MI: University of Michigan Press, 1975.

[10] D. Goldberg, Genetic Algorithms in Search, Optimization, and Machine Learning. New York: Addison-Wesley, 1989.

[11] K. A. De Jong, Analysis of Behavior of a Class of Genetic Adaptive Systems. PhD thesis, University of Michigan, Ann Arbor, MI, 1975.

[12] K. A. De Jong, "Adaptive system design: A genetic approach," IEEE Transaction Sys. Man. Cybern., vol. 10, no. 3, pp. 556-574, 1980

[13] K. De Jong, "Learning with genetic algorithms: An overview," Machine Learning, no. 3, pp. 121-138, 1988

[14] A. Sheta and K. D. Jong, "Parameter estimation of nonlinear systems in noisy environment using genetic algorithms," in Proceedings of the IEEE International Symposium on Intelligent Control (ISIC'96), pp. 360-366, 1996.

[15] D. E. Goldberg, The Design of Innovation: Lessons from and for Competent Genetic Algorithms. Boston, MA: Kluwer Academic, 2002.

[16] K. DeJong, An Analysis of Behavior of a Class of Genetic Adaptive Systems, Doctoral dissertation. $\mathrm{PhD}$ thesis, University of Michigan, Dissertation Abstracts International, 1975.

[17] D. E. Goldberg, Computer-Aided Gas Pipeline Operation Using Genetic Algorithms and Rule Learning. $\mathrm{PhD}$ thesis, University of Michigan, Ann Arbor, 1983.

[18] J. Lee, R. Haralick, and L. Shapiro, "Morphologic edge detection," J-RA, vol. 3, pp. 142-156, 1987.

[19] V. di Gesu and G. lo Bosco, "Image segmentation based on genetic algorithms combination," in CIAP05, pp. 352-359, 2005.

[20] J. Ruisz, D. Honigmann, and H. Pottmann, "Segmentation and modeling of approximately rotationally symmetric objects in $3 \mathrm{~d}$ ultrasound," in 3DIM03, pp. 124-131, 2003.

[21] R. Ohlander, K. Price, and D. R. Reddy, "Picture segmentation using a recursive region splitting method," Computer Graphs and Image Processing, vol. 8, pp. 313-333, 1978. 